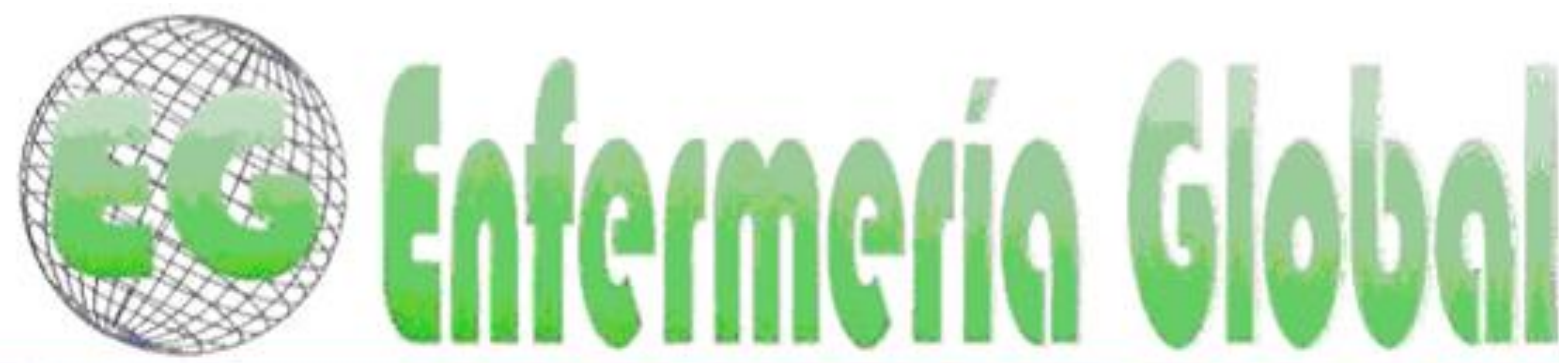

ISSN 1695-6141

Revista electrónica trimestral de Enfermeria

No34

Abril 2014

www.um.es/egloball

REVISIONES

\title{
Errores de medicación en el ejercicio de la enfermería: una revisión integrativa
}

Erro de medicação no exercício da enfermagem: uma revisão integrativa

Medication error in the exercise of nursing: an integrative review

\section{${ }^{*}$ Franco da Silva, Eveline *de Faveri, Fabiano *Lorenzini, Elisiane \\ *Enfermera/o. Docente del curso de Enfermería. Facultad Nossa Senhora de Fátima. Caxias do Sul, R/S. Brasil. E-mail: evelinefranco@yahoo.com.br}

Palabras clave: errores de medicación; enfermería; gestión de la seguridad

Palavras-chave: erros de medicação; enfermagem; gerenciamento de segurança

Keywords: medication errors; nursing; safety management.

\section{RESUMEN}

Este estudio tiene como objetivo analizar las publicaciones nacionales sobre errores de medicación en el ejercicio de la enfermería. Es una revisión integradora, realizada en las bases de datos BDEnf, SciELO y LILACS. Del análisis se seleccionaron cuatro temas: el conocimiento de los criterios que caracterizan a los errores de medicación,causas que originan el error; punición cuando se produce un error, y educación continua como herramienta para reducir los errores de medicación. Se encontró que responsabilizar al profesional por el error es una cultura que persiste en Brasil.

\section{RESUMO}

Estudo com objetivo de analisar as publicações nacionais sobre erro de medicação no exercício de enfermagem. Trata-se de uma revisão integrativa, realizada por meio das bases de dados BDEnf, SciELO e LILACS. Da análise foram elencados quatro temas: conhecimento dos critérios que caracterizam o erro de medicação; causas que originam o erro; punição na ocorrência de erro; e educação continuada como ferramenta para redução do erro de medicação. Observou-se que responsabilizar o profissional pelo erro é uma cultura que persiste no Brasil.

\section{ABSTRACT}

This study is aimed at analyzing the national publications about medication error in the exercise of nursing. This is an integrative review, conducted by searching the databases BDEnf, SciELO and LILACS. Four topics emerged from the analysis: knowledge of the criteria that characterize the medication error, the error causes; punishment when an error occurs and continuing education as a tool 
to reduce medication errors. We found that the professional responsibility for the mistake is a culture that persists in Brazil.

\section{INTRODUCCIÓN}

La Agencia Nacional de Vigilancia Sanitaria define el error de medicación como cualquier evento evitable que puede causar o inducir el uso inapropiado de medicamento o perjudicar al paciente en cuanto que el medicamento está bajo control del profesional de salud, paciente o consumidor. ${ }^{(1)}$

Tales eventos tienen que ver con la práctica profesional, productos de salud, procedimientos y sistemas, seguimiento y uso de los medicamentos. En Brasil, un paciente ingresado es víctima de, por lo menos, un error de medicación al día ${ }^{(1,2)}$

El compromiso ético con las acciones de cuidado de enfermería se justifica con los nuevos modelos de administración que defienden la calidad. Partiendo de dicha defensa, la ocurrencia de hechos iatrogénicos en las acciones de enfermería contraría tales preceptos, ya que se pone en riesgo la seguridad del usuario de los servicios de salud y, consecuentemente, la calidad de asistencia ${ }^{(3)}$

En el ejercicio de la enfermería, la preparación y la administración de medicamentos es parte de la carga de trabajo, de esa manera, la clase profesional está sujeta a cometer errores. Frente a esto, una cuestión inquietante es: ¿cómo enfermería investiga sus posibles errores de medicación y las consecuencias que estos traen para enfermería? Por lo tanto, el objetivo del estudio ha sido analizar las publicaciones nacionales sobre errores de medicación en el ejercicio de la enfermería.

\section{MÉTODO}

Se trata de una revisión integradora, método de investigación que permite la incorporación de las evidencias en la práctica clínica con el fin de reunir y sintetizar resultados de investigación sobre dicho tema o cuestión, de forma sistemática y ordenada ${ }^{(4)}$

Para la elaboración de este estudio, con base en la metodología de revisión integradora ${ }^{(4)}$, se cumplieron las etapas: formulación de la pregunta de investigación; recolección de datos; evaluación, análisis e interpretación de datos; presentación de los resultados.

Para guiar esta investigación se formuló la sigiuiente pregunta: ¿cómo enfermería investiga sus posibles errores de medicación y las implicaciones derivadas de ellos para enfermería?

En el periodo de agosto a diciembre de 2011, se buscó en las bases de datos BDEnf, SciElo y LILACS utilizando los siguientes descriptores: error de medicación AND enfermería y respetando los criterios de inclusión: artículos originarios de estudios realizados en Brasil, con publicación entre 2000 y 2011, idioma portugués, que tuvieran informaciones sobre la actuación de enfermería en errores de medicación. Para el análisis y síntesis de los artículos se construyó un recuadro sinóptico, que contenía los aspectos considerados pertinentes: nombre del artículo; área de concentración; objetivos; resultados y consideraciones/conclusiones. 
Se identificaron 53 publicaciones y tras análisis minucioso la muestra seleccionó 18 artículos.

Los datos utilizados en este estudio fueron debidamente referenciados, respetando e identificando a sus autores y demás fuentes de investigación, observando el rigor ético sobre la propiedad intelectual de los textos científicos que fueron investigados, en lo que se refiere al uso del contenido y de citación de las partes de las obras consultadas. Con la finalidad de mantener la evaluación ciega, los artículos fueron codificados (A.01, A.02,..., A.18), solamente después del análisis fueron identificados los autores.

\section{RESULTADOS}

Los artículos incluidos en este estudio (Cuadro 01) permearon los diversos periódicos del área de enfermería, solamente un artículo fue publicado en periódico destinado a las ciencias biomédicas y de la salud.

\begin{tabular}{|l|l|l|l|}
\hline CÓDIGO & \multicolumn{1}{|c|}{ TítULO AÑO } & PERIÓDICO \\
\hline A.01 & $\begin{array}{l}\text { Administração endovenosa de antibióticos e } \\
\text { resistência bacteriana: responsabilidade da } \\
\text { enfermagem }\end{array}$ & 2006 & $\begin{array}{l}\text { Rev Eletr } \\
\text { Enfermagem }\end{array}$ \\
\hline A.02 & $\begin{array}{l}\text { Análise dos comportamentos dos profissionais de } \\
\text { enfermagem frente aos erros na administração de } \\
\text { medicamentos }\end{array}$ & 2002 & $\begin{array}{l}\text { Acta Paul } \\
\text { Enferm }\end{array}$ \\
\hline A.03 & $\begin{array}{l}\text { Conduta do futuro enfermeiro mediante o erro de } \\
\text { medicação }\end{array}$ & 2009 & $\begin{array}{l}\text { Rev UFPE } \\
\text { On line } \\
\text { (REUOL) }\end{array}$ \\
\hline A.04 & $\begin{array}{l}\text { Consequências de medicação em unidades de } \\
\text { terapia intensiva e semi-intensiva }\end{array}$ & 2006 & $\begin{array}{l}\text { Rev Esc Enf } \\
\text { USP }\end{array}$ \\
\hline A.05 & $\begin{array}{l}\text { Erro de medicação: importância da notificação no } \\
\text { gerenciamento da segurança do paciente }\end{array}$ & 2007 & $\begin{array}{l}\text { Rev Bras } \\
\text { Enferm }\end{array}$ \\
\hline A.06 & $\begin{array}{l}\text { Erros de medicação: condutas e propostas de } \\
\text { prevenção na perspectiva da equipe de enfermagem }\end{array}$ & 2007 & $\begin{array}{l}\text { Rev Eletr } \\
\text { Enferm }\end{array}$ \\
\hline A.07 & $\begin{array}{l}\text { Erros na medicação e consequências para } \\
\text { profissionais de enfermagem e clientes: um estudo } \\
\text { exploratório }\end{array}$ & 2002 & $\begin{array}{l}\text { Rev Latino- } \\
\text { am Enferm }\end{array}$ \\
\hline A.08 & $\begin{array}{l}\text { Eventos adversos com medicação em serviços de } \\
\text { emergência: condutas profissionais e sentimentos } \\
\text { vivenciados por enfermeiros }\end{array}$ & 2005 & $\begin{array}{l}\text { Rev Bras } \\
\text { Enferm }\end{array}$ \\
\hline A.09 & $\begin{array}{l}\text { O sistema de medicação nos hospitais e sua } \\
\text { avaliação por um grupo de profissionais }\end{array}$ & 2005 & $\begin{array}{l}\text { Rev Esc Enf } \\
\text { USP }\end{array}$ \\
\hline A.10 & $\begin{array}{l}\text { Percepções sobre o erro de medicação: análise de } \\
\text { respostas da equipe de enfermagem }\end{array}$ & 2006 & $\begin{array}{l}\text { Rev Latino- } \\
\text { am Enferm }\end{array}$ \\
\hline A.11 & $\begin{array}{l}\text { Segurança na terapia medicamentosa: uma revisão } \\
\text { bibliográfica }\end{array}$ & 2008 & $\begin{array}{l}\text { Esc } \\
\text { Nery Rna } \\
\text { enferm Rev }\end{array}$ \\
\hline A.12 & $\begin{array}{l}\text { Sentimentos de profissionais de enfermagem após a } \\
\text { ocorrência de erros de medicação }\end{array}$ & 2007 & $\begin{array}{l}\text { Acta Paul } \\
\text { enferm }\end{array}$ \\
\hline $\begin{array}{l}\text { Segurança do paciente: análise do preparo de } \\
\text { medicação intravenosa em hospital da rede } \\
\text { sentinela }\end{array}$ & 2011 & $\begin{array}{l}\text { Texto } \\
\text { Contexto } \\
\text { Enferm }\end{array}$ \\
\hline
\end{tabular}




\begin{tabular}{|l|l|c|l|}
\hline A.14 & $\begin{array}{l}\text { Condutas adotadas por técnicos de enfermagem } \\
\text { após ocorrência de erros de medicação }\end{array}$ & 2010 & $\begin{array}{l}\text { Acta Paul } \\
\text { Enferm }\end{array}$ \\
\hline A.15 & $\begin{array}{l}\text { Avaliação dos fatores de risco relacionados às } \\
\text { falhas durante a administração de medicamentos }\end{array}$ & 2008 & $\begin{array}{l}\text { Arq Ciênc } \\
\text { Saúde } \\
\text { Unipar }\end{array}$ \\
\hline A.16 & $\begin{array}{l}\text { Estratégias para prevenção de erros de medicação } \\
\text { no Setor de Emergência }\end{array}$ & 2005 & $\begin{array}{l}\text { Rev Bras } \\
\text { Enferm }\end{array}$ \\
\hline A.17 & $\begin{array}{l}\text { Erros de medicação: análise do conhecimento da } \\
\text { equipe de enfermagem de uma instituição hospitalar }\end{array}$ & 2011 & $\begin{array}{l}\text { Rev Gau } \\
\text { Enferm }\end{array}$ \\
\hline A.18 & $\begin{array}{l}\text { Erros e ações praticadas pela instituição hospitalar } \\
\text { no preparo e administração de medicamentos }\end{array}$ & 2011 & $\begin{array}{l}\text { REME Rev } \\
\text { Min Enferm }\end{array}$ \\
\hline
\end{tabular}

Con relación al tipo de publicación se observó que la mayoría consistía en artículos originales (obtenidos por investigación). El abordaje más usado en las investigaciones fue cuantitativo de cuño descriptivo, solamente tres estudios usaron el abordaje cualitativo (A.02, A.12 e A.14) y dos estudios eran revisión de la literatura.

Del análisis de las producciones salieron las categorías: conocimiento de los criterios que caracterizaron el error de medicación; causas que originan el error; punición en la ocurrencia del error; y formación continua como herramienta para la reducción del error de medicación.

\section{DISCUSIÓN DE LOS DATOS}

\section{Conocimiento de los criterios que caracterizan el error de medicación}

En esta categoría se identificó la comprensión de los profesionales sobre los criterios que caracterizan el error de medicación. Se percibió que hay algunas controversias también evidenciadas en el estudio que muestra el uso de diversos vocablos y significados diferentes para el mismo hecho ocurrido, lo que puede constituir un factor de confusión.

Los errores son clasificados como errores de omisión intencional y no intencional y errores de autoridad intencional y no intencional. Errores de omisión intencional involucran la falla de propósito al administrar un medicamento prescrito a un paciente que no debe recibirlo. Errores de omisión no intencional son los que ocurren cuando una medicación no prescrita es deliberadamente administrada a un paciente, mientras que el no intencional, es cuando un paciente, accidentalmente, recibe una medicación prescrita de forma incorrecta. Ambos errores involucran fallos al administrar el remedio prescrito ${ }^{(6)}$

En el estudio A.03, 77 (68\%) de los encuestados mencionó que es necesario mejorar el conocimiento sobre el tema. Cuando les fue preguntado sobre el significado de la expresión error de medicación, 73 (65\%) dijeron que no sabrían identificar un error de medicación y 40 (35\%) respondieron que no ${ }^{(7)}$.

Se piensa que las instituciones deben desarrollar e implementar programas de educación centrados en los principios generales de seguridad del paciente y que incluyan informaciones sobre todos los tipos de posibles errores, como por ejemplo: error de prescripción, error de dispensación, error de horario, error de administración no autorizada de medicamento, error de dosis, error de presentación, error de 
preparación, error de administración, error con medicamentos caducados y error de seguimiento..

\section{Causas que dan origen al error}

En esta categoría se evidencian las múltiples causas que conducen al error de medicación. Es importante señalar que el error no siempre se origina en la actividad del profesional de enfermería. En la investigación que analizó 4.026 prescripciones, se hizo evidente que hay un $89,1 \%$ de problemas de cuatro tipos: ausencia de concentración y forma farmacéutica, poca legibilidad y concentración dudosa ${ }^{(8)}$.

El estudio A.04, cuyo objetivo fue percibir los errores en una muestra de 50 pacientes, obtuvo un total de 52 errores identificados, de ellos 23,08\% ocurrieron por omisión de dosis, $21,15 \%$ e $17,31 \%$ por medicamentos y dosis equivocadas, respectivamente ${ }^{(9)}$

Estudios muestran variación elevada en las tasas de errores originados en la preparación de medicamentos ${ }^{(2,10)}$. Se considera que el medicamento es un instrumento y que el enfermero debe estar comprometido con los resultados de su uso. Las prácticas gerenciales deben garantizar procesos más seguros en el manejo de los medicamentos ${ }^{(2)}$.

Las muchas circunstancias que llevan a errores de horario, retraso, error en la manipulación de bombas de infusión continua y omisión en la administración de una medicación deben ser analizadas. Pueden ser causadas por falta de medicación en la unidad, un gran número de ítemes para administrar, muchos pacientes ingresados, demora en la dispensación de los remedios, hasta un dimensionamiento inadecuado de personal ${ }^{(11)}$.

En el estudio A.02, los autores dicen que las instituciones deben mantener ambientes seguros con disponibilidad de recursos humanos y físicos, como elementos necesarios para la prevención de futuros errores de medicación ${ }^{(6)}$. En esa perspectiva, se entiende que el uso de tecnología, con prescripciones y dispensaciones de medicamentos completamente automatizadas, se constituye en importante factor de reducción del número de errores.

\section{Punición en la ocurrencia del error}

En esta categoría, se evidencia la ocurrencia de la punición como factor contribuyente para la notificación del error. Medidas administrativas como advertencia verbal, notificación de la ocurrencia, advertencia escrita y dimisión han sido tomadas como punición del profesional involucrado en el error ${ }^{(6)}$.

Pero, la existencia del miedo a sufrir punición, la dimisión, el sentimiento de culpa y las inquietudes relacionadas con la gravedad del error pueden llevar al individuo involucrado a no notificar el error, estando el grado de penalización del profesional involucrado en relación con la gravedad de las lesiones corporales ${ }^{(7,12)}$; además de eso, en algunos casos, puniciones aplicadas al profesional involucrado en el error son registradas en los documentos internos del profesional bajo custodia de la guardia del sector de administración de personal ${ }^{(12)}$

Sobre las medidas tomadas por la institución con el profesional involucrado en el error, el estudio A.09 relata que la mayoría (78\%) de los participantes están de 
acuerdo con estas decisiones, de esos $31,8 \%$ defienden que el profesional debe responder por sus actos y actitudes mencionando también el déficit de atención, conocimiento y poca concentración como algunos de los factores contribuyentes al error en la medicación ${ }^{(13)}$.

Todavía se observa la defensa de la aplicación de medidas no punitivas, haciendo de ello una palanca para que los profesionales cuenten los errores, dándoles la oportunidad de revisión de los procesos de trabajo y con esto, mayor seguridad a la asistencia del paciente ${ }^{(10)}$.

El enfoque de que actos inseguros, entre ellos el error de medicación, es un proceso mental de olvido y ausencia de atención, conduce a tratarlos con medidas disciplinares, culpa y humillación, siendo que el problema de este abordaje es que, focalizando al profesional como causa del error, se aíslan los actos inseguros del contexto sistémico, recayendo en patrones recurrentes ${ }^{(14)}$

Frente a esta realidad, se entiende que hay una necesidad de trabajar la implementación de otras estrategias sustitutivas a la punición, estrategias que busquen analizar la causa del error de medicación, con énfasis en la visión del proceso y no solamente en la persona, posibilitando la minimización real de dichas ocurrencias dentro de las instituciones de salud.

\section{Formación continua como herramienta para la reducción del error de medicación}

Se observa la importancia de estrategias de formación continua como factor de impacto en la reducción de ocurrencia de errores de medicación. Conocimiento insuficiente y poca experiencia también han sido identificados como factores contribuyentes, lo que apunta al factor humano como causante de los errores ${ }^{(15)}$

En un estudio realizado con estudiantes de enfermería, se evidencia que gran parte de éstos afirma ser necesario desarrollar el conocimiento en relación a los errores de medicación y relató que, en la ocurrencia de errores de medicamentos no intencionales, optaría por la orientación del equipo ${ }^{(7)}$.

La formación continua, a través de capacitaciones profesionales, puede ser usada como estrategia frente al error de medicación $(12,16,17)$. Estudios muestran la necesidad de uso de estrategias de educación en el área de farmacología, dirigidas a todo el equipo relacionado con la cadena de medicamentos dentro de las instituciones $^{(2,16)}$, siendo necesario el énfasis en el aprendizaje para evitar la reincidencia de los errores ${ }^{(12)}$. Se sabe que el desarrollo de los profesionales, a través de capacitaciones sobre errores de medicamentos, es una de las sugerencias más referidas como estrategia para cambiar la ocurrencia de ellos, seguida por redoblar la atención ${ }^{(18)}$. En ese sentido, se refuerza la necesidad de invertir en la mejora de los procesos de trabajo, instituyéndose programas de desarrollo de los profesionales en ese tema, para posibilitar condiciones para la minimización de los errores de medicamentos.

\section{CONCLUSIONES}

Esa revisión permitió una mayor visibilidad a los estudios nacionales sobre errores de medicamentos en el ejercicio de enfermería, permitiendo identificar que el uso de 
vocablos y significados diferentes para el mismo hecho, permite a los profesionales el no reconocimiento de dichos eventos como errores de medicación.

Los estudios indican que responsabilizar al profesional del error es una cultura persistente en Brasil.

Se evidencia la necesidad de invertir en formación continua asociada a la informatización de los procesos asistenciales, como estrategia para la reducción sustancial de esos errores.

Pese a una creciente concienciación, aún es necesario avanzar mucho para conseguir tales patrones de calidad en atención a la salud en Brasil.

Se observa que la mayoría de las investigaciones posee carácter cuantitativo. De ahí la importancia de desarrollar estudios cualitativos sobre el tema que puedan indicar nuevas estrategias para mejoras en el proceso del cuidado de enfermería previniendo la ocurrencia de errores.

\section{REFERENCIAS}

1. Brasil. Resolução RDC no 04 de 10 de fevereiro de 2009. Dispõe sobre as normas de farmacovigilância para detentores de registro de medicamentos de uso humano; 2009.

2. Camerini FG, Silva LD. Segurança do paciente: análise do preparo de medicação intravenosa em hospital da rede sentinela. Texto Contexto Enferm. 2011;20(1):41-9. 3. Cecchetto FH, Fachinelli TS, Souza EN. latrogenic or adverse event: perception of nursing staff. Rev Enferm UFPE On Line [periódico na internet]. $2010 \mathrm{Jul} / \mathrm{Set}$ [acesso em 2011 nov 07]; 4(3):1377-38. Disponível em: http://www.ufpe.br/revistaenfermagem/index.php/revista/article/view/985.

4. Mendes KDS, Silveira RCCP, Galvão CM. Revisão integrativa: método de pesquisa para a incorporação de evidências na saúde e na enfermagem. Texto Contexto Enferm. 2008;17(4):758-64.

5. Madalosso ARM. latrogenia do cuidado de enfermagem: dialogando com o perigo no quotidiano profissional. Rev Latino-Am Enfermagem. 2000;8:11-7.

6. Carvalho VT, Cassiani SH. Análise dos comportamentos dos profissionais de enfermagem frente aos erros na administração de medicamentos. Acta Paul Enferm. 2002;15(2):45-54.

7. Pereira CMB, Pereira OB, Carboni RM. Conduct of the future nurses in the perspective of medication error. Rev Enferm UFPE On Line [periódico na internet]. 2010 Jan/Mar [acesso em 2011 nov 07]; 4(1):118-23. Disponível em:

http://www.ufpe.br/revistaenfermagem/index.php/revista/article/view/536/pdf 301.

8. Freire CC, Gimenes FRE, Cassiani SHDB. Análise da prescrição informatizada em duas clínicas de um hospital universitário. Medicina (Ribeirão Preto). 2004;37(1/2):916.

9. Toffoletto MC, Padilha KG. Conseqüências de medicação em unidades de terapia intensiva e semi-intensiva. Rev Esc Enferm USP. 2006;40(2):247-52.

10. Bohomol E, Ramos LH. Erro de medicação: importância da notificação no gerenciamento da segurança do paciente. Rev Bras Enferm. 2007;60(16):32-6.

11. Smetzer J. Take 10 giant steps to medication safety: CE Connection Nursing; 2001 [acesso em 05 mai 2002]. Disponível em: http://www.springnet.com. 
12. Silva BK, Silva JS, Gobbo AFF, Miasso AI. Erros de medicação: condutas e propostas de prevenção na perspectiva da equipe de enfermagem. Rev Eletr Enf. 2007;9(3):712-23.

13. Cassiani SHDB, Teixeira TCA, Opitz SP, Linhares JC. O sistema de medicação nos hospitais e sua avaliação por um grupo de profissionais. Rev Esc Enferm USP. 2005;39(3):280-7.

14. Cassiani SHDB. A segurança do paciente e o paradoxo no uso de medicamentos. Rev Bras Enferm. 2005;58(1):95-9.

15. Praxedes MFS, Filho PCPT. Erros e ações praticadas pela instituição hospitalar no preparo e administração de medicamentos. REME Rev Min Enferm.

2011;15(3):406-11.

16. Oliveira RC, Camargo AEB, Cassiani SHDB. Estratégias para prevenção de erros de medicação no Setor de Emergência. Rev Bras Enferm. 2005;58(4):399-404.

17. Freitas DF, Oda JY. Avaliação dos fatores de risco relacionados às falhas durante a administração de medicamentos. Arq ciências saúde UNIPAR. 2008;12(3):231-7.

18. Carvalho VT, Cassiani SHDB, Chiericato C, Miasso Al. Rev Latino-am Enferm.

Erros mais comuns e fatores de risco na administração de medicamentos em unidades básicas de saúde. 1999;7(5):67-75. 